\title{
Algorithm Analysis of Numerical Solutions to the Heat Equation
}

\author{
Edmund Agyeman \\ Department of Mathematics, \\ Kwame Nkrumah University of Science and \\ Technology, Kumasi-Ghana.
}

\author{
Derick Folson \\ Department of Mathematics, \\ Kwame Nkrumah University of Science and \\ Technology, Kumasi-Ghana.
}

\begin{abstract}
The numerical algorithms employed in the solution of Parabolic Partial Differential Equations are the subject of this paper. In particular, the Crank-Nicolson scheme, which is generally accepted as an improvement of the Schmidt scheme, is subjected not only to stability analysis, but also absolute relative error analysis to guide Mathematicians and Engineers alike to know the true performance of these numerical solution methods. The Heat Equation $T_{t}=c T_{x x}$ with Dirichlet conditions conducting heat is analysed by employing the analytical method of solution where the method of Separation of Variables is used. The same equation is then solved with the Schmidt scheme as well as the Crank-Nicolson scheme and the results compared to the analytical solution.

It is shown that provided stability conditions for both numerical schemes are not compromised, the Schmidt scheme is better than the Crank-Nicolson scheme at the particular point $80 \%$ from the conducting end of the rod. With the rod discretized into six points, both ends of the rod produce the same results for both numerical schemes. With the remaining four points, it is shown that three points produced values which showed that the Crank-Nicolson scheme is better than the Schmidt scheme at those three points, but not the fourth.
\end{abstract}

\section{General Terms}

Numerical Solution, Finite Difference, Relative Absolute Error, Parabolic PDEs, Separation of Variables, Dirichlet condition, Crank-Nicolson scheme, Schmidt scheme, Stability

\section{Keywords}

Numerical Analysis, Algorithm, Heat Equation

\section{INTRODUCTION}

Numerical mathematics has come to the aid of mathematicians for centuries and has made the solution of otherwise unsolvable mathematical problems quite easy. This has been felt in almost all branches of Science especially, mathematics, engineering and medicine.

When numerical mathematics reached its peak in the mid $20^{\text {th }}$ century, among those who made their names were the German scholar Erhard Schmidt (forming the Schmidt method), English Mathematical mathematicians John Crank and Phyllis Nicolson (together forming the Crank-Nicolson method). These outstanding mathematicians of old proposed algorithms for solving partial differential equations numerically.

Even though these algorithms are used widely today in almost all fields of Science, time has come to put these algorithms under the lens for informed decision to be made on them. Although quite some steps have been taken by various mathematicians to analyse these algorithms, all effort unfortunately focus only on their stability analysis.

\section{OBJECTIVES}

This paper among other things seeks to:

- Apply analytical and numerical methods to solve a parabolic partial differential equation.

- Compare the numerical solutions to the analytical solution and draw informed conclusions about the numerical algorithms.

- Compare and contrast error analysis and stability analysis.

\section{METHODOLOGY}

A model problem for an aluminium rod of length L, initially at room temperature with one end immersed in boiling water and the other insulated is considered. The subsequent temperature distribution across the rod is computed analytically as well as numerically. Numerically, two algorithms are employed to compare and contrast their performance in terms of efficiency and accuracy:

- Schmidt Scheme

- Crank-Nicolson Scheme

The solutions will be limited by the following conditions so as to achieve uniformity in solution.

$$
0 \leq x \leq 1 \quad 0 \leq t \leq 0.1
$$

Computer algebra systems will be employed to minimize errors if not eradicate it completely.

\section{JUSTIFICATION}

Heat equation has many applications in engines and structural mechanics. It is also used extensively in Biology where it is known as diffusion equation and models the diffusion of substances such as drugs, bacterial, or viral spread in the human system. This research paper will put to rest the overreliance on stability concepts alone in selecting appropriate numerical algorithms for predicting the behaviour of heat transfer by the heat equation. This will also help pharmacists in predicting the behaviour of certain drugs in the human body. It will also put in retrospect which numerical scheme is best for solving parabolic PDEs in general.

\section{ANALYTI CAL SOLUTION}

Let $T(x, t)$ be the temperature in degrees Celsius at a distance $x \mathrm{ft}$ from the hot end, $t$ minutes after the end at $x=0$ is immersed. Let $c$ be the thermal diffusivity of the rod. The temperature is governed by the problem

$$
\begin{array}{cc}
T_{t}=c T_{x x} & 0 \leq x \leq L, \\
T(0, t)=100, & T_{x}(L, t)=0, \\
T(x, 0)=20
\end{array}
$$

This problem is not correctly formulated for the separation of variables technique because the boundary condition at $x=0$ 
is non-homogenous. Observe that the temperature profile as $t \rightarrow \infty$ is easily determined by setting $T_{t}$ equal to 0 and ignoring the initial condition. The result is the steady-state temperature problem

$$
T_{s}^{\prime \prime}=0, \quad T_{s}(0)=100, \quad T_{s}^{\prime}(L)=0,
$$

where $T_{S}(x)$ is the steady-state temperature. The solution of this simple problem is the constant $T_{s} \equiv 100$. Suppose we subtract $T_{S}$ from $T$ to obtain a new temperature variable, say

$$
\omega=T-T_{S}=T-100
$$

with $\omega$ in place of $T$, the problem becomes

$$
\begin{array}{cc}
\omega_{t}=c \omega_{x x} & 0 \leq x \leq L, \\
\omega(0, t)=0, & \omega_{x}(L, t)=0, \\
\omega(x, 0)=-80 &
\end{array}
$$

The new problem has homogenous boundary conditions. Simplifying the problem by scaling, a new temperature and time is defined by

$$
u=\frac{\omega}{80}=\frac{T-100}{80}, \quad \tau=c t
$$

With these changes, the problem becomes

$$
\begin{array}{cr}
u_{\tau}=u_{x x} & 0 \leq x \leq L, \\
u(0, \tau)=0 & , u_{x}(L, \tau)=0, \\
u(x, 0)=-1
\end{array}
$$

To solve the above problem, the first thing to do is to apply separation of variables. First, assume that the solution takes the form

Substituting

$$
u(x, t)=\varphi(x) G(\tau)
$$

$$
\begin{gathered}
\Rightarrow \frac{d}{d \tau}(\varphi(x) G(\tau))=\frac{d^{2}}{d x^{2}}(\varphi(x) G(\tau)) \\
\varphi(x) \frac{d G}{d \tau}=G(\tau) \frac{d^{2} G}{d x^{2}}
\end{gathered}
$$

Separating the variables gives

$$
\frac{1}{G} \frac{d G}{d \tau}=\frac{1}{\varphi} \frac{d^{2} \varphi}{d x^{2}}
$$

The only condition that will make the above equation meaningful is when both sides evaluate to a constant. ie

$$
\frac{1}{G} \frac{d G}{d \tau}=\frac{1}{\varphi} \frac{d^{2} \varphi}{d x^{2}}=-\lambda
$$

where $-\lambda$ is called the separation constant and is arbitrary.

The equation above can be split it into the following two ordinary differential equations

$$
\frac{d G}{d \tau}=-G \lambda, \quad \frac{d^{2} \varphi}{d x^{2}}=-\varphi \lambda
$$

The next step is to make sure that the product solution, $u(x, \tau)=\varphi(x) G(\tau)$, satisfies the boundary conditions so it is plugged into both expressions

$$
u(0, \tau)=\varphi(0) G(\tau)=0 \quad u_{x}(L, x)=\varphi(0) G(\tau)=0
$$

If the first equation is considered, it is either $\omega(0)=0$ or $G(\tau)=0$. However $G(\tau)=0$ for every $\tau$ and $u(x, \tau)=0$, ie the trivial solution, and a solution to any linear homogeneous equation, a non-trivial solution is more desirable. Therefore it is assumed that infact $\varphi(0)=0$.

Likewise from the second, $\varphi^{\prime}(L)=0$ to avoid the trivial solution. In summary

$$
\begin{array}{cc}
\frac{d G}{d \tau}=-\lambda G, \quad \frac{d^{2} \varphi}{d x^{2}}+\lambda G=0 \\
\varphi(0)=0 & \varphi^{\prime}(L)=0
\end{array}
$$

and note that there is not a condition for the time differential equation and that is not a problem.
The time dependent equation can really be solved at any time, but since $\lambda$ is yet unknown, let's hold on. Now the spatial problem solution is

$$
\begin{gathered}
\frac{d^{2} \varphi}{d x^{2}}+\lambda G=0 \\
\varphi(0) \stackrel{=}{=} \quad \varphi^{\prime}(L)=0
\end{gathered}
$$

There are three possible scenarios to deal with here.

CASE 1: $\lambda>0$

In this case the solution to the differential equation is

$$
\varphi(x)=c_{1} \cos (\sqrt{\lambda} x)+c_{2} \sin (\sqrt{\lambda} x)
$$

Applying the first boundary condition gives

$$
0=\varphi(0)=c_{1}
$$

Applying the second boundary condition and using the immediate result yields

$$
0=\varphi^{\prime}(L)=c_{2} \cos (L \sqrt{\lambda})
$$

Going after non-trivial solutions means

$$
\cos (L \sqrt{\lambda})=0 \Rightarrow L \sqrt{\lambda}=\frac{(2 n-1) \pi}{2} n=1,2,3, \ldots
$$

Note that $c_{2}$ is not needed in the eigenfunction as it will get absorbed into another constant that will be picked up later on.

\section{CASE 2: $\lambda=0$}

The solution to the differential equation is

$$
\varphi(x)=c_{1}+c_{2} x
$$

Applying the boundary condition gives

$$
0=\varphi(0)=c_{1} \quad 0=\varphi^{\prime}(L)=c_{2} L \quad \Rightarrow c_{2}=0
$$

So in this case the only solution is the trivial solution and so $\boldsymbol{\lambda}=\mathbf{0}$ is not an eigenvalue for this boundary value problem.

\section{CASE 3: $\lambda<0$}

Here the solution to the differential equation is

$$
\varphi(x)=c_{1} \cosh (\sqrt{\lambda} x)+c_{2} \sinh (\sqrt{\lambda} x)
$$

Applying the first homogenous condition gives

$$
0=\varphi(0)=c_{1}
$$

and applying the second gives

$$
0=\varphi^{\prime}(L)=c_{2} \cosh (L \sqrt{-\lambda})
$$

Assuming $\quad \lambda<0$ and so $\quad \boldsymbol{L} \sqrt{-\lambda} \neq \mathbf{0}$ and this means $\cosh (\boldsymbol{L} \sqrt{-\lambda}) \neq \mathbf{0}$. Thus, $\boldsymbol{c}_{\mathbf{2}}=\mathbf{0}$ is the only trivial solution in this case. Therefore there will be no negative eigenvalues for this boundary value problem.

The complete list of eigenvalues and eigenfunctions for this problem are then

$$
\lambda_{n}=\left(\frac{(2 n-1) \pi}{2 L}\right)^{2} \varphi_{n}(x)=\sin \left(\frac{(2 n-1) \pi x}{2 L}\right), n=1,2, \ldots
$$

Now solving the time differential equation

$$
\frac{d G}{d \tau}=-\lambda G
$$

and note that because of simplicity, $\lambda$ is not substituted.

This is a simple linear (and separable for that matter) $1^{\text {st }}$ order differential equation and the solution is

$$
G(\tau)=h e^{-\lambda_{n} \tau}=h e^{-\left(\frac{(2 n-1) \pi}{2 L}\right)^{2}} \quad n=1,2,3, \ldots
$$

Now that both the ordinary and partial differential equations are solved a final solution can be written. Note however the 
infinitely many solutions found since there are infinitely many solutions (ie eigenfunctions) to the spatial problem. The product solutions are then

$$
u(x, t)=B_{n} \sin \left(\frac{(2 n-1) \pi x}{2 L}\right) e^{-\left(\frac{(2 n-1) \pi}{2 L}\right)^{2} \tau} \quad n=1,2, \ldots
$$

The product solution is denoted $\boldsymbol{u}_{\boldsymbol{n}}$ to acknowledge that each value of $n$ will yield a different solution. Also note that the $h$ in the solution to the time problem is changed to $\boldsymbol{B}_{\boldsymbol{n}}$ to denote the fact that it will probably be different for each value of $n$ as well and because had the $\boldsymbol{c}_{2}$ been kept with the specific eigenfunction it would have absorbed the $c$ to get a single constant in the solution.

The principle of Superposition is not restricted to only two solutions and so the following is also a solution to the partial differential equation

$$
u(x, t)=\sum_{n=1}^{M} B_{n} \sin \left(\frac{(2 n-1) \pi x}{2 L}\right) e^{-\left(\frac{(2 n-1) \pi}{2 L}\right)^{2} \tau}
$$

Extending the solution further by taking $M \rightarrow \infty$ yields

$$
u(x, t)=\sum_{n=1}^{\infty} B_{n} \sin \left(\frac{(2 n-1) \pi x}{2 L}\right) e^{-\left(\frac{(2 n-1) \pi}{2 L}\right)^{2} \tau}
$$

The solution for the coefficient $B_{n}$ is given by

$$
B_{n}=\frac{2}{L} \int_{0}^{L} f(x) \sin \left(\frac{(2 n-1) \pi x}{2 L}\right) d x \quad n=1,2,3, \ldots
$$

where $f(x)=u(x, 0)$. By setting $L=1$ and evaluating $B_{n}$ yields

Hence

$$
B_{n}=-\frac{4}{(2 n-1) \pi}
$$

$u(x, t)=\sum_{n=1}^{\infty}-\frac{4}{(2 n-1) \pi} \sin \left(\frac{(2 n-1) \pi x}{2 L}\right) e^{-\left(\frac{(2 n-1) \pi}{2 L}\right)^{2} \tau}$

Recovering the original solution and completely eliminating $\mathrm{L}$ by substitution,

$$
\begin{aligned}
& T(x, t)=100+80 u(x, k t) \\
& =100+80 \sum_{n=1}^{\infty}-\frac{4}{(2 n-1) \pi} \sin \left(\frac{(2 n-1) \pi x}{2 L}\right) e^{-\left(\frac{(2 n-1) \pi}{2 L}\right)^{2} k \tau} \\
& =100-\frac{320}{\pi} \sum_{n=1}^{\infty}-\frac{1}{(2 n-1)} \sin \left(\frac{(2 n-1) \pi x}{2 L}\right) e^{-\left(\frac{(2 n-1) \pi}{2 L}\right)^{2} k \tau}
\end{aligned}
$$

Table 1. Solution of the Heat Equation Using the Analytical method

\begin{tabular}{|c|l|l|l|l|l|l|}
\hline & $\boldsymbol{x}_{\boldsymbol{A 1}}=\mathbf{0 . 0 0}$ & $\boldsymbol{x}_{\boldsymbol{A} \mathbf{2}}=\mathbf{0 . 2 0}$ & $\boldsymbol{x}_{\boldsymbol{A} \mathbf{3}}=\mathbf{0 . 4 0}$ & $\boldsymbol{x}_{\boldsymbol{A 4}}=\mathbf{0 . 6 0}$ & $\boldsymbol{x}_{\boldsymbol{A} \mathbf{}}=\mathbf{0 . 8 0}$ & $\boldsymbol{x}_{\boldsymbol{A} \mathbf{6}}=\mathbf{1 . 0 0}$ \\
\hline$t_{1}=0.00$ & 100.0000 & 41.0591 & 7.8616 & 7.1536 & 23.1400 & 32.1212 \\
\hline$t_{2}=0.01$ & 100.0000 & 47.3001 & 15.7528 & 11.2460 & 21.5185 & 27.8412 \\
\hline$t_{3}=0.02$ & 100.0000 & 52.4317 & 22.3267 & 14.8744 & 20.6334 & 24.8485 \\
\hline$t_{4}=0.03$ & 100.0000 & 56.6716 & 27.8398 & 18.1226 & 20.3277 & 22.8759 \\
\hline$t_{5}=0.04$ & 100.0000 & 60.1944 & 32.4973 & 21.0581 & 20.4761 & 21.7098 \\
\hline$t_{6}=0.05$ & 100.0000 & 63.1399 & 36.4637 & 23.7349 & 20.9787 & 21.1795 \\
\hline$t_{7}=0.06$ & 100.0000 & 65.6200 & 39.8711 & 26.1968 & 21.7553 & 21.1483 \\
\hline$t_{8}=0.07$ & 100.0000 & 67.7245 & 42.8253 & 28.4787 & 22.7424 & 21.5072 \\
\hline$t_{9}=0.08$ & 100.0000 & 69.5255 & 45.4109 & 30.6089 & 23.8890 & 22.1691 \\
\hline$t_{10}=0.09$ & 100.0000 & 71.0805 & 47.6961 & 32.6104 & 25.1546 & 23.0645 \\
\hline$t_{11}=0.10$ & 100.0000 & 72.4358 & 49.7355 & 34.5014 & 26.5072 & 24.1379 \\
\hline
\end{tabular}




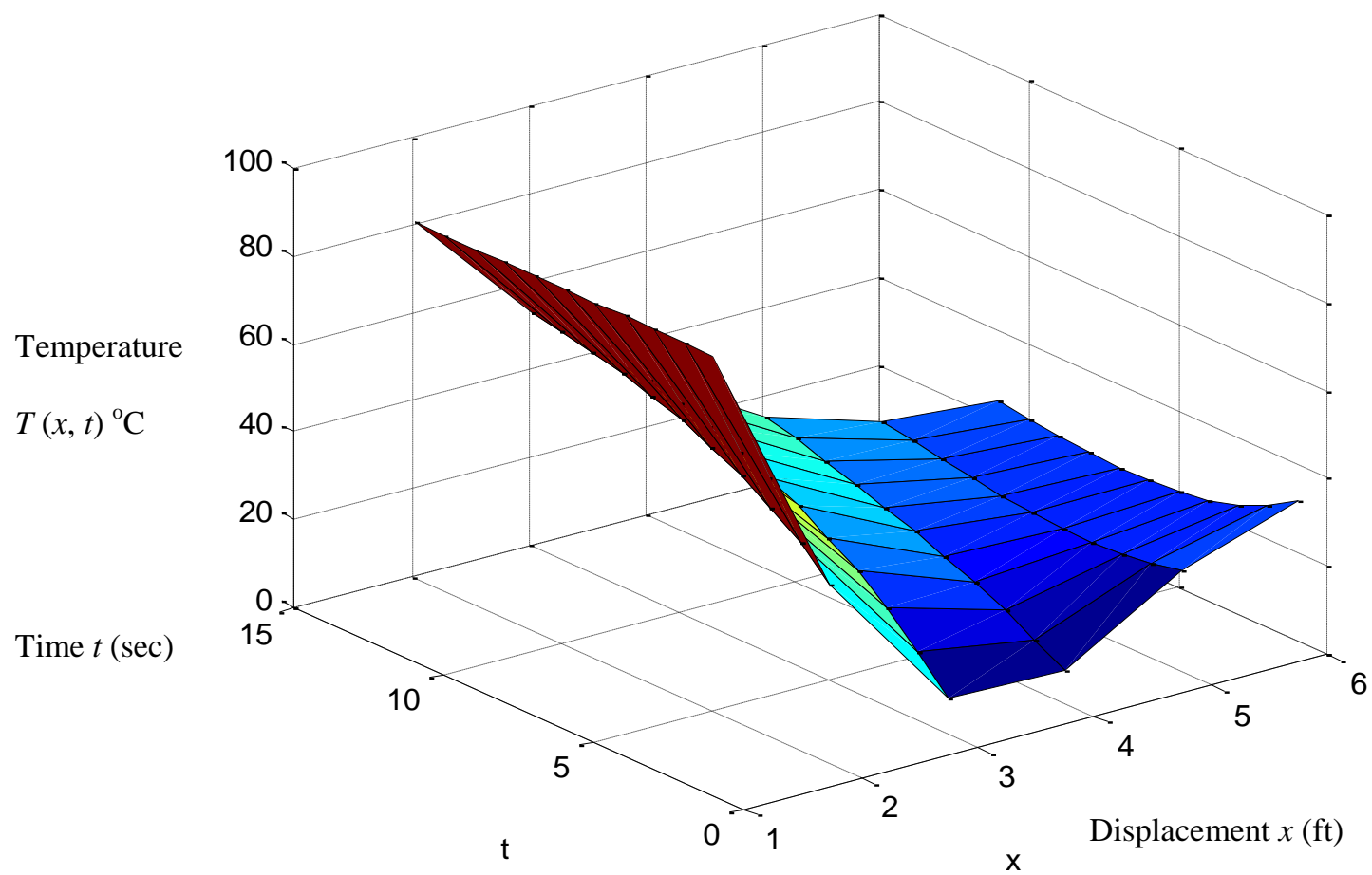

Figure 1: A 3D-Plot of the data produced in the Analytical solution

\section{NUMERICAL SOLUTION OF THE HEAT EQUATION}

To solve the heat equation numerically, both the $x$ and $t$ variables need to be discretized and proceed to deal with the $x$-variable employing finite difference approximation. This concept according to these great schools of thought Schmidt and Crank-Nicolson is presented in this paper.

\subsection{Schmidt Scheme}

When Dirichlet boundary conditions are imposed, those values must be specified at the boundary points. The firstorder forward-time second-order centred-space (FTSC) approximation of the heat equation is given by

$$
U_{i}^{j+1}=r U_{i-1}^{j}+(1-2 r) U_{i}^{j}+r U_{i+1}^{j}
$$

\subsubsection{Stability of Schmidt Scheme Using Von} Neumann/Fourier Method

$$
\begin{gathered}
U_{i}^{j+1}=r U_{i-1}^{j}+(1-2 r) U_{i}^{j}+r U_{i+1}^{j}+R_{E n, m+1} \\
\bar{U}_{i}^{j+1}=r \bar{U}_{i-1}^{j}+(1-2 r) \bar{U}_{i}^{j}+r \bar{U}_{i+1}^{j}+T_{E n, m+1} \\
\quad \text { Let } \boldsymbol{\varepsilon}_{\boldsymbol{n}}^{\boldsymbol{m}}=\boldsymbol{U}_{\boldsymbol{i}}^{j}-\overline{\boldsymbol{U}}_{\boldsymbol{i}}^{j}
\end{gathered}
$$

(1)-(2) $\Rightarrow \varepsilon_{n}^{m+1}-r \varepsilon_{n-1}^{m}-(1-2 r) \varepsilon_{n}^{m}-r \varepsilon_{n+1}^{m}=$ $R_{E n, m+1}-T_{E n, m+1}$

Von Neumann considers the homogenous part of the difference equation

$\Rightarrow \varepsilon_{n}^{m+1}-r \varepsilon_{n-1}^{m}-(1-2 r) \varepsilon_{n}^{m}-r \varepsilon_{n+1}^{m}=0$

Using Fourier series, the n-component solution of the difference scheme is

$$
\varepsilon_{n}^{m}=c e^{\delta m k+1, i \beta n h}=c e^{\delta m k} e^{i \beta n h}
$$

Substituting (4) in (3) and simplifying yields

$$
e^{\delta k}=1-2 r+\left(e^{i \beta h}+e^{-i \beta h}\right)
$$

With the following trigonometric identities

$$
\cos \theta=\frac{1}{2}\left(e^{i \theta}+e^{-i \theta}\right)=1-2 \sin ^{2} \frac{\theta}{2}
$$

Equation (5) then becomes

$$
e^{\delta k}=1-4 r \sin ^{2} \frac{\beta h}{2}
$$

For the original error not to grow, the amplification factor is restricted as $\left|e^{\delta k}\right| \leq 1$

$$
\Rightarrow\left|1-4 r \sin ^{2} \frac{\beta h}{2}\right| \leq 1
$$

As sine function has the range $-\mathbf{1} \leq \sin \theta \leq \mathbf{1}$

$$
\begin{gathered}
\Rightarrow-1 \leq\left|1-4 r \sin ^{2} \frac{\beta h}{2}\right| \leq 1 \\
\Leftrightarrow-2 \leq\left|-4 r \sin ^{2} \frac{\beta h}{2}\right| \leq 0
\end{gathered}
$$

But $\max \left|\sin ^{2} \frac{\beta h}{2}\right|=1$

$$
\Rightarrow-2 \leq-4 r<0 \Rightarrow 0<4 r \leq 2 \quad \therefore 0<r \leq
$$

$\frac{1}{2}$

This confirms that provided $0<r \leq \frac{1}{2}$ then the Schmidt scheme will be stable, otherwise it will be unstable. In other words, the Schmidt scheme is conditionally stable.

For the purpose of this paper, $r$ is set to 0.5 to achieve stability. The table below shows the resulting computations using the Schmidt scheme. 
Table 2. Solution of the Heat Equation using the Schmidt Scheme

\begin{tabular}{|l|l|l|l|l|l|l|}
\hline & $\boldsymbol{x}_{\boldsymbol{S 1}}=\mathbf{0 . 0 0}$ & $\boldsymbol{x}_{\boldsymbol{S} \mathbf{2}}=\mathbf{0 . 2 0}$ & $\boldsymbol{x}_{\boldsymbol{S 3}}=\mathbf{0 . 4 0}$ & $\boldsymbol{x}_{\boldsymbol{S 4}}=\mathbf{0 . 6 0}$ & $\boldsymbol{x}_{\boldsymbol{S} \mathbf{5}}=\mathbf{0 . 8 0}$ & $\boldsymbol{x}_{\boldsymbol{S} \mathbf{6}}=\mathbf{1 . 0 0}$ \\
\hline$t_{1}=0.00$ & 100.0000 & 20.0000 & 20.0000 & 20.0000 & 20.0000 & 20.0000 \\
\hline$t_{2}=0.01$ & 100.0000 & 40.0000 & 20.0000 & 20.0000 & 20.0000 & 20.0000 \\
\hline$t_{3}=0.02$ & 100.0000 & 50.0000 & 25.0000 & 20.0000 & 20.0000 & 20.0000 \\
\hline$t_{4}=0.03$ & 100.0000 & 56.2500 & 30.0000 & 21.2500 & 20.0000 & 20.0000 \\
\hline$t_{5}=0.04$ & 100.0000 & 60.6250 & 34.3750 & 23.1250 & 20.3125 & 20.0000 \\
\hline$t_{6}=0.05$ & 100.0000 & 63.9063 & 38.1250 & 25.2344 & 20.9375 & 20.0000 \\
\hline$t_{7}=0.06$ & 100.0000 & 66.4844 & 41.3477 & 27.3828 & 21.7773 & 20.0000 \\
\hline$t_{8}=0.07$ & 100.0000 & 68.5791 & 44.1406 & 29.4727 & 22.7344 & 20.0000 \\
\hline$t_{9}=0.08$ & 100.0000 & 70.3247 & 46.5833 & 31.4551 & 23.7354 & 20.0000 \\
\hline$t_{10}=0.09$ & 100.0000 & 71.8082 & 48.7366 & 33.3072 & 24.7314 & 20.0000 \\
\hline$t_{11}=0.10$ & 100.0000 & 73.0882 & 50.6471 & 35.0206 & 25.6925 & 20.0000 \\
\hline
\end{tabular}

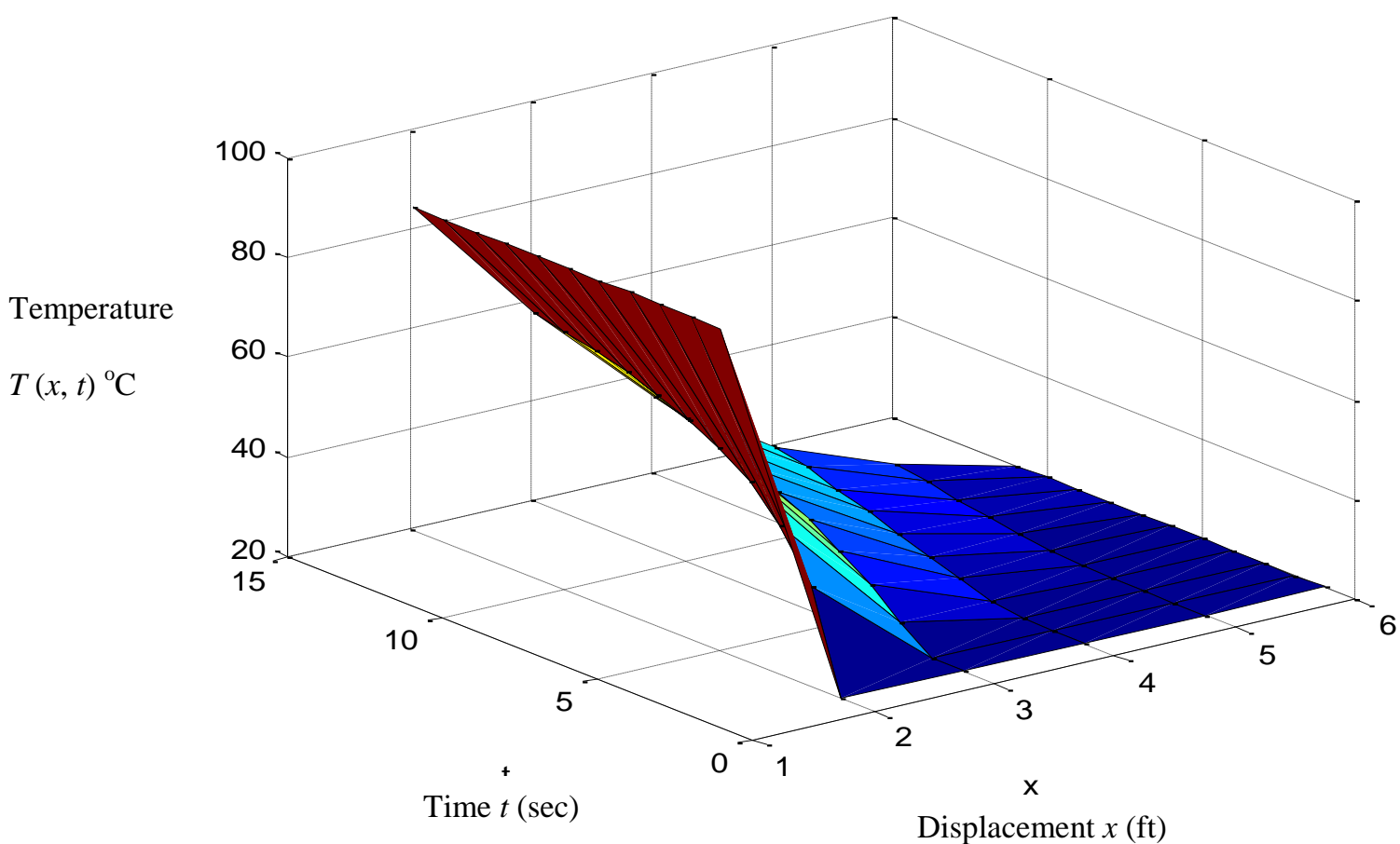

Figure 2: A 3D-Plot of the data produced by the Schmidt scheme with $r=0.5$

\subsection{Cranch Nicolson Scheme}

$$
\begin{aligned}
-r U_{i-1}^{j+1}+2(1+r) & U_{i}^{j+1}-r U_{i-1}^{j+1} \\
& =r U_{i-1}^{j}+2(1-r) U_{i}^{j}+r U_{i+1}^{j}
\end{aligned}
$$

6.2.1 Stability Analysis of the Crank-Nicolson Scheme Using Von Neumann/Fourier Method

$$
\begin{gathered}
-r U_{i-1}^{j+1}+2(1+r) U_{i}^{j+1}-r U_{i-1}^{j+1} \\
=r U_{i-1}^{j}+2(1-r) U_{i}^{j}+r U_{i+1}^{j} \\
-r \bar{U}_{i-1}^{j+1}+2(1+r) \bar{U}_{i}^{j+1}-r \bar{U}_{i-1}^{j+1} \\
=r \bar{U}_{i-1}^{j}+2(1-r) \bar{U}_{i}^{j}+r \bar{U}_{i+1}^{j}
\end{gathered}
$$

Let $\varepsilon_{n}^{m}=U_{i}^{j}-\bar{U}_{i}^{j}$

$$
\begin{array}{r}
(a)-(b) \stackrel{r}{\Rightarrow}-r \varepsilon_{n-1}^{m+1}+2(1+r) \varepsilon_{n}^{m+1}-r \varepsilon_{n-1}^{m} \\
\quad=r \varepsilon_{n-1}^{m}+2(1-r) \varepsilon_{n}^{m}+r \varepsilon_{n+1}^{m}
\end{array}
$$

Using Fourier series, the n-component solution of the difference scheme is

$$
\varepsilon_{n}^{m}=c e^{\delta m k+1, i \beta n h}=c e^{\delta m k} e^{i \beta n h}
$$

Substituting (d) in (c) and simplifying yields

$$
\begin{aligned}
& e^{\delta k}\left[2(1+r)-r\left(e^{i \beta h}+e^{-i \beta h}\right)\right] \\
& \quad=2(1-r)+r\left(e^{i \beta h}+e^{-i \beta h}\right)
\end{aligned}
$$

With the following trigonometric identities

$$
\cos \theta=\frac{1}{2}\left(e^{i \theta}+e^{-i \theta}\right)=1-2 \sin ^{2} \frac{\theta}{2}
$$

Equation (f) then becomes

$$
e^{\delta k}=\frac{2-4 r \sin ^{2} \frac{\beta h}{2}}{2+4 r \sin ^{2} \frac{\beta h}{2}}
$$

Guided by the fact that $\left|e^{\delta k}\right| \leq 1$ and $\max \left|\sin ^{2} \frac{\beta h}{2}\right|=1$ 
$\Rightarrow\left|\frac{2-4 r \sin ^{2} \frac{\beta h}{2}}{2+4 r \sin ^{2} \frac{\beta h}{2}}\right| \leq 1 \quad \Leftrightarrow\left|\frac{2-4 r}{2+4 r}\right| \leq 1$
This shows that the Crank-Nicolson scheme is unconditionally stable but for the sake of uniformity and comparison, $r=0.5$ is chosen. The result of the CrankNicolson scheme computation is shown in the table below:

Table 3. Solution of the Heat Equation Using the Crank-Nicolson Scheme

\begin{tabular}{|l|l|l|l|l|l|l|}
\hline & \multicolumn{1}{|c|}{$\boldsymbol{x}_{\mathbf{1}}=\mathbf{0 . 0 0}$} & \multicolumn{1}{|c|}{$\boldsymbol{x}_{\mathbf{2}}=\mathbf{0 . 2 0}$} & \multicolumn{1}{|c|}{$\boldsymbol{x}_{\mathbf{3}}=\mathbf{0 . 4 0}$} & \multicolumn{1}{|c|}{$\boldsymbol{x}_{\mathbf{4}}=\mathbf{0 . 6 0}$} & $\boldsymbol{x}_{\mathbf{5}}=\mathbf{0 . 8 0}$ & \multicolumn{1}{c|}{$\boldsymbol{x}_{\mathbf{6}}=\mathbf{1 . 0 0}$} \\
\hline$t_{1}=0.00$ & 100.0000 & 20.0000 & 20.0000 & 20.0000 & 20.0000 & 0.0000 \\
\hline$t_{2}=0.01$ & 100.0000 & 36.1612 & 21.6122 & 19.9608 & 17.9961 & 20.0000 \\
\hline$t_{3}=0.02$ & 100.0000 & 46.3832 & 25.2524 & 20.3454 & 18.8283 & 20.0000 \\
\hline$t_{4}=0.03$ & 100.0000 & 53.2854 & 29.3022 & 21.4936 & 19.4809 & 20.0000 \\
\hline$t_{5}=0.04$ & 100.0000 & 58.2207 & 33.1921 & 23.1086 & 20.1487 & 20.0000 \\
\hline$t_{6}=0.05$ & 100.0000 & 61.9253 & 36.737 & 24.9626 & 20.8964 & 20.0000 \\
\hline$t_{7}=0.06$ & 100.0000 & 64.8192 & 39.9033 & 26.9036 & 21.7244 & 20.0000 \\
\hline$t_{8}=0.07$ & 100.0000 & 67.1532 & 42.7133 & 28.8372 & 22.6087 & 20.0000 \\
\hline$t_{9}=0.08$ & 100.0000 & 69.0838 & 45.2061 & 30.7071 & 23.5197 & 20.0000 \\
\hline$t_{10}=0.09$ & 100.0000 & 70.7131 & 47.4223 & 32.4821 & 24.4307 & 20.0000 \\
\hline$t_{11}=0.10$ & 100.0000 & 72.1100 & 49.3985 & 34.1466 & 25.3213 & 20.0000 \\
\hline
\end{tabular}

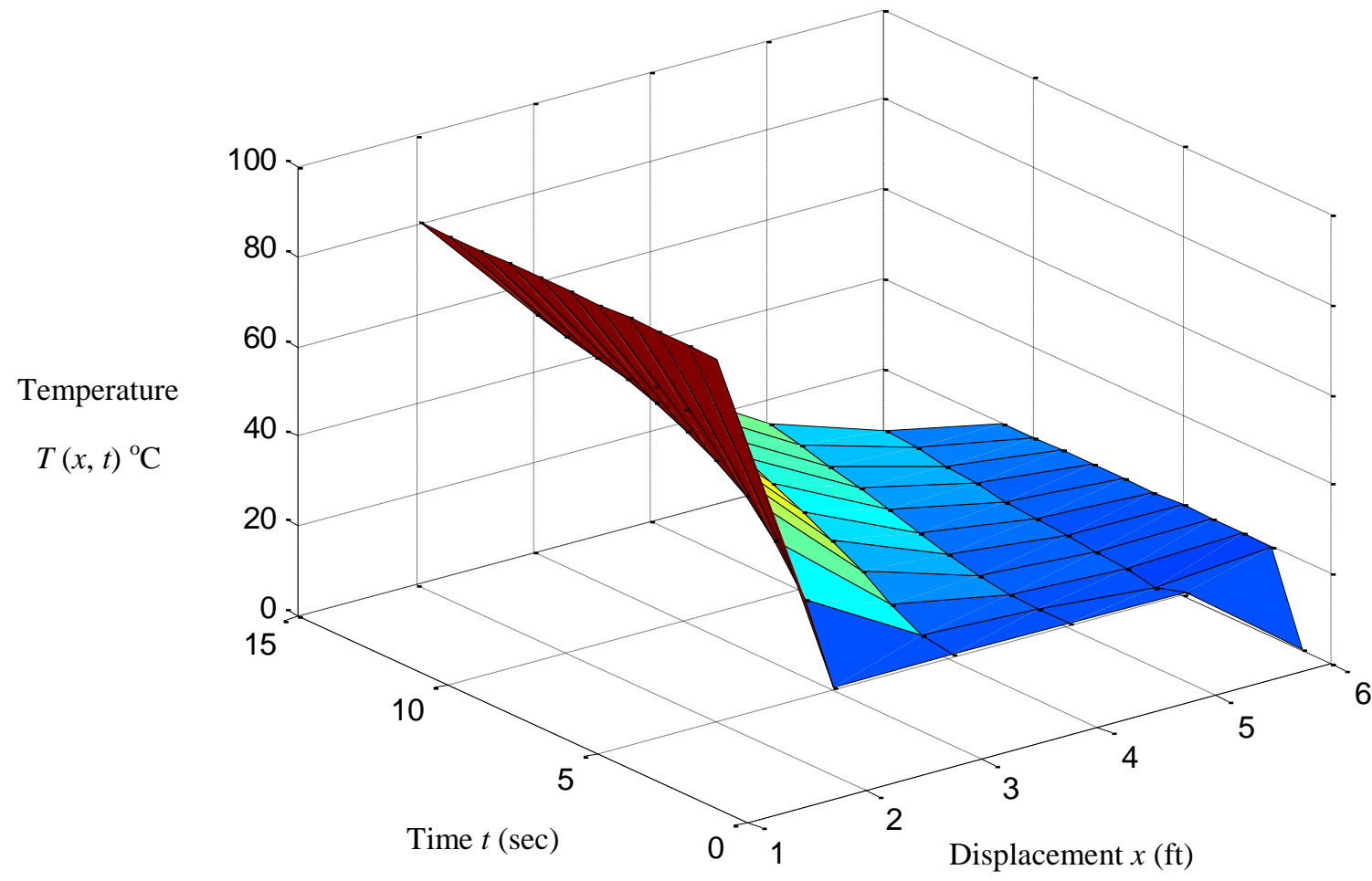

Figure 3: A 3D-Plot of the data produced in the Crank-Nicolson scheme with $\boldsymbol{r}=0.5$. 
7. DATA ANALYSIS

Table 4. Relative Errors in Numerical Approximations Using the Schmidt Scheme

\begin{tabular}{|c|c|c|c|c|c|c|}
\hline & $\frac{\left|\boldsymbol{x}_{(\boldsymbol{A}-\boldsymbol{S}) \mathbf{1}}\right|}{\left|\boldsymbol{x}_{\boldsymbol{A} 1}\right|}$ & $\frac{\left|\boldsymbol{x}_{(\boldsymbol{A}-\boldsymbol{S}) \mathbf{2}}\right|}{\left|\boldsymbol{x}_{\boldsymbol{A} 2}\right|}$ & $\frac{\left|\boldsymbol{x}_{(\boldsymbol{A}-\boldsymbol{S}) \mathbf{3}}\right|}{\left|\boldsymbol{x}_{\boldsymbol{A 3}}\right|}$ & $\frac{\left|\boldsymbol{x}_{(\boldsymbol{A}-\boldsymbol{S}) \mathbf{4}}\right|}{\left|\boldsymbol{x}_{\boldsymbol{A 4}}\right|}$ & $\frac{\left|\boldsymbol{x}_{(\boldsymbol{A}-\boldsymbol{S}) \mathbf{5}}\right|}{\left|\boldsymbol{x}_{\boldsymbol{A} 5}\right|}$ & $\frac{\left|\boldsymbol{x}_{(\boldsymbol{A}-\boldsymbol{S}) \mathbf{6}}\right|}{\left|\boldsymbol{x}_{\boldsymbol{A 6}}\right|}$ \\
\hline$t_{1}=0.00$ & 0.0000 & 0.5129 & 1.5440 & 1.7958 & 0.1357 & 0.3774 \\
\hline$t_{2}=0.01$ & 0.0000 & 0.1543 & 0.2696 & 0.7784 & 0.0706 & 0.2816 \\
\hline$t_{3}=0.02$ & 0.0000 & 0.0464 & 0.1197 & 0.3446 & 0.0307 & 0.1951 \\
\hline$t_{4}=0.03$ & 0.0000 & 0.0074 & 0.0776 & 0.1726 & 0.0161 & 0.1257 \\
\hline$t_{5}=0.04$ & 0.0000 & 0.0072 & 0.0578 & 0.0982 & 0.0080 & 0.0788 \\
\hline$t_{6}=0.05$ & 0.0000 & 0.0121 & 0.0456 & 0.0632 & 0.0020 & 0.0557 \\
\hline$t_{7}=0.06$ & 0.0000 & 0.0132 & 0.0370 & 0.0453 & 0.0010 & 0.0543 \\
\hline$t_{8}=0.07$ & 0.0000 & 0.0126 & 0.0307 & 0.0349 & 0.0004 & 0.0701 \\
\hline$t_{9}=0.08$ & 0.0000 & 0.0115 & 0.0258 & 0.0276 & 0.0064 & 0.0978 \\
\hline$t_{10}=0.09$ & 0.0000 & 0.0102 & 0.0218 & 0.0214 & 0.0168 & 0.1329 \\
\hline$t_{11}=0.10$ & 0.0000 & 0.0090 & 0.0183 & 0.0150 & 0.0307 & 0.1714 \\
\hline
\end{tabular}

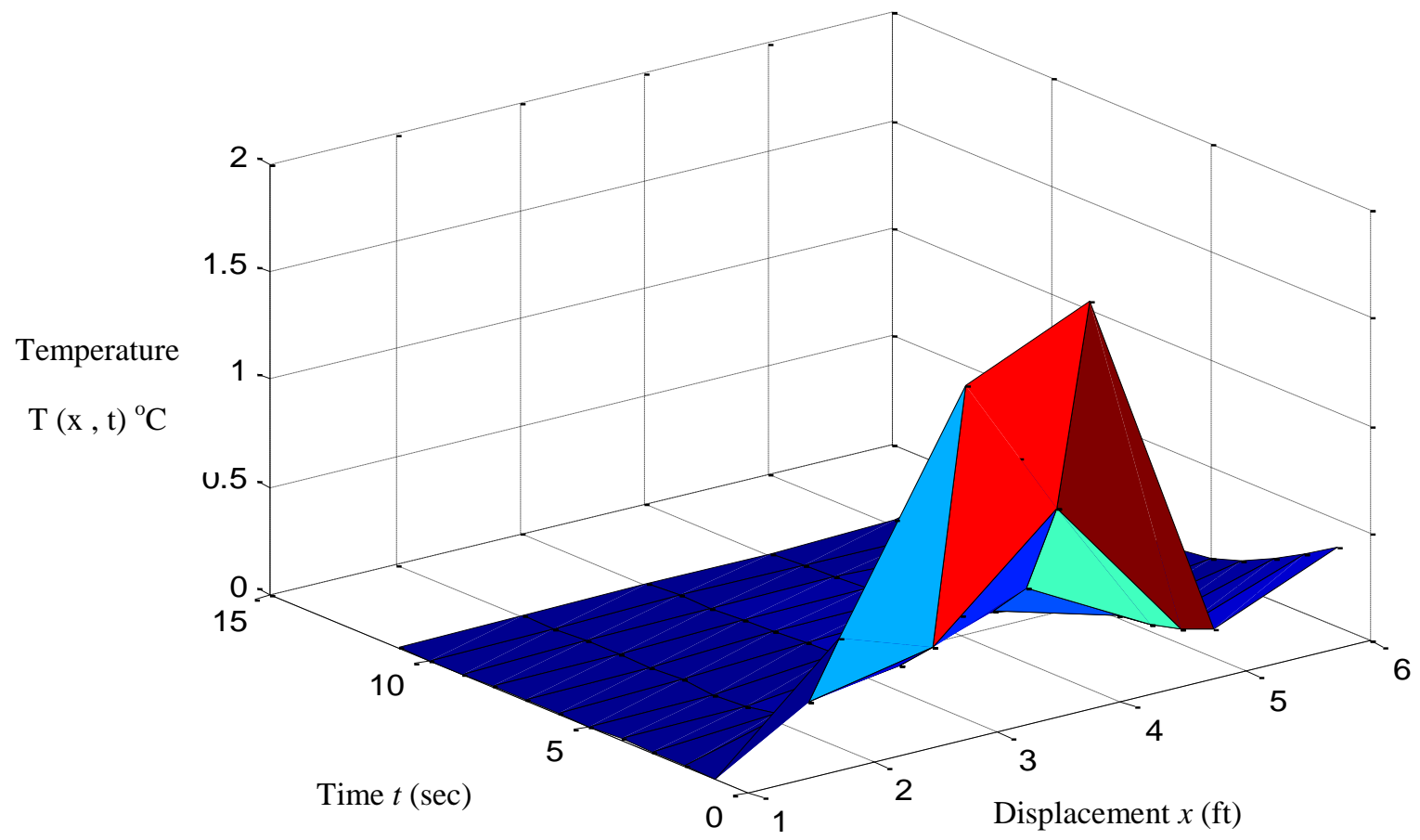

Figure 4: A 3D-Plot of the absolute relative error with the Schmidt scheme

Table 5. Relative Errors in Numerical Approximations Using the Crank-Nicolson Scheme

\begin{tabular}{|c|c|c|c|c|c|c|}
\hline & $\frac{\left|x_{(A-C N) 1}\right|}{\left|x_{A 1}\right|}$ & $\frac{\left|x_{(A-C N) 2}\right|}{\left|x_{A 2}\right|}$ & $\frac{\left|x_{(A-C N) 3}\right|}{\left|x_{A 3}\right|}$ & $\frac{\left|x_{(A-C N) 4}\right|}{\left|x_{A 4}\right|}$ & $\frac{\left|x_{(A-C N) 5}\right|}{\left|x_{A 5}\right|}$ & $\frac{\left|x_{(A-C N) 6}\right|}{\left|x_{A 6}\right|}$ \\
\hline$t_{1}=0.00$ & 0.0000 & 0.5129 & 1.5440 & 1.7958 & 0.1357 & 1.0000 \\
\hline$t_{2}=0.01$ & 0.0000 & 0.2355 & 0.3720 & 0.7749 & 0.1637 & 0.2816 \\
\hline$t_{3}=0.02$ & 0.0000 & 0.1154 & 0.1310 & 0.3678 & 0.0875 & 0.1951 \\
\hline$t_{4}=0.03$ & 0.0000 & 0.0598 & 0.0525 & 0.1860 & 0.0417 & 0.1257 \\
\hline$t_{5}=0.04$ & 0.0000 & 0.0328 & 0.0214 & 0.0974 & 0.0160 & 0.0788 \\
\hline$t_{6}=0.05$ & 0.0000 & 0.0192 & 0.0075 & 0.0517 & 0.0039 & 0.0557 \\
\hline$t_{7}=0.06$ & 0.0000 & 0.0122 & 0.0008 & 0.0270 & 0.0014 & 0.0543 \\
\hline$t_{8}=0.07$ & 0.0000 & 0.0084 & 0.0026 & 0.0126 & 0.0059 & 0.0701 \\
\hline$t_{9}=0.08$ & 0.0000 & 0.0064 & 0.0045 & 0.0032 & 0.0155 & 0.0978 \\
\hline$t_{10}=0.09$ & 0.0000 & 0.0052 & 0.0057 & 0.0039 & 0.0288 & 0.1329 \\
\hline$t_{11}=0.10$ & 0.0000 & 0.0045 & 0.0068 & 0.0103 & 0.0447 & 0.1714 \\
\hline
\end{tabular}




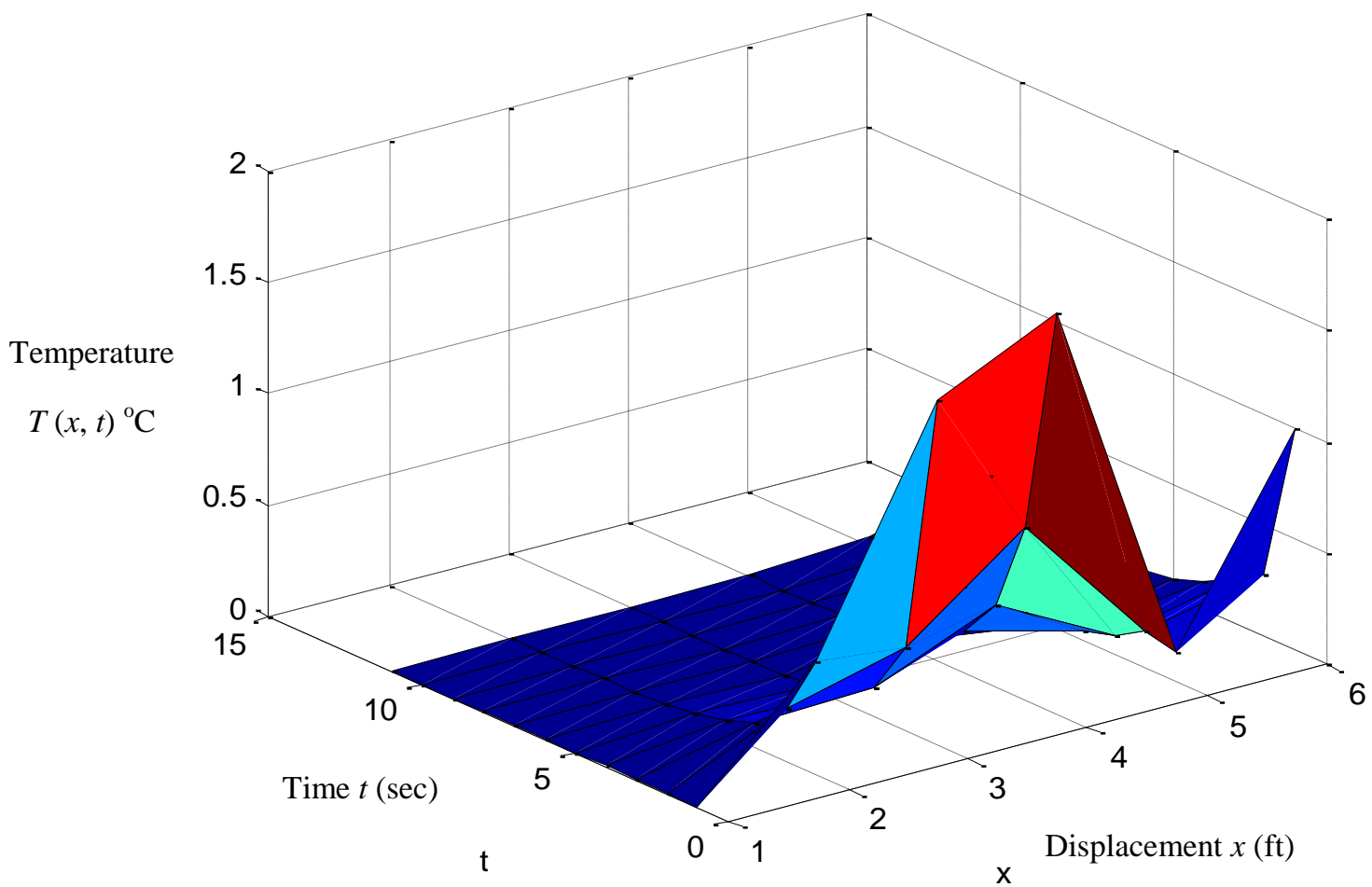

Figure 5: A 3D Plot of the Absolute Relative Error with the Crank-Nicolson scheme.

\section{DISCUSSION OF RESULTS}

For an aluminium rod at room temperature whose one end is insulated and the other immersed in boiling water, one would expect that with time the temperature will grow uniformly through the rod until such a time that the temperature distribution is the same across the whole rod. Even though, the rod was not given enough time to undergo such a transformation, that is, with in a time span of $0 \leq t \leq 0.1$, it was still evidently clear that the temperature distribution was actually growing across the rod.

For the analytical solution, the temperature distribution at $0.4 \leq x \leq 0.6$, for $0 \leq t \leq 0.01$, fell below the room temperature of the rod, which was quite unusual. It begun to improve when $0.02 \leq t \leq 0.03$, and only at $x=0.6$ when temperature actually fell below room temperature. For $0 \leq t \leq 0.05$ and $x=0.8$ something unusual was happening. All the temperature at that point was actually less than the insulated end of the rod.

The aluminium rod begun to exhibit its true conductivity characteristics from $t=0.06$, when there was truly uniformity in heat transfer.

The Schmidt scheme conforms to initial conditions of the rod perfectly. Heat only begins to flow at $t=0.01$ when the temperature doubled at the point $x=0.2$ while other parts of the rod remained at room temperature. The temperature gradually increases through the rod until $t=0.04$ when all parts of the rod had experience temperature rise. This time conforms to the time the rod exhibits its conductivity properties.

The initial conditions of the rod is perfectly obeyed by the Crank-Nicolson method with the exception that $(1,0)=0$. This defeats the fact that $T(x, 0)=20$. This initial condition is however satisfied from $t=0.01$ whiles $0.6 \leq x \leq 0.8$ experiences a decrease in temperature. This negative phenomenon decreases in the range $0.02 \leq t \leq 0.03$. The conductivity property of the rod then starts from $t=0.04$.

Generally, the values obtained with the Schmidt and the Crank-Nicolson schemes compare favourably with values obtained with the analytical method of solution

$T(x, t)=100-\frac{320}{\pi} \sum_{n=1}^{\infty} \frac{1}{(2 n-1)} \sin \left(\frac{(2 n-1) \pi x}{2}\right) e^{-\left(\frac{(2 n-1) \pi}{2}\right)^{2} k t}$

. The true value for the final solution for the Analytical, Schmidt, and Crank-Nicolson schemes is shown in Table 6; 
Table 6. True Values of the Various Methods to the Heat Equatio

\begin{tabular}{|l|c|l|l|l|l|l|l|}
\hline & & $\boldsymbol{x}_{\mathbf{1}}=\mathbf{0 . 0 0}$ & $\boldsymbol{x}_{\mathbf{2}}=\mathbf{0 . 2 0}$ & $\boldsymbol{x}_{\mathbf{3}}=\mathbf{0 . 4 0}$ & $\boldsymbol{x}_{\mathbf{4}}=\mathbf{0 . 6 0}$ & $\boldsymbol{x}_{\mathbf{5}}=\mathbf{0 . 8 0}$ & $\boldsymbol{x}_{\mathbf{6}}=\mathbf{1 . 0 0}$ \\
\hline Analytical Scheme & $t_{11}$ & 100.0000 & 72.4358 & 49.7355 & 34.5014 & 26.5072 & 24.1379 \\
\hline Schmidt Scheme & $t_{11}$ & 100.0000 & 73.0882 & 50.6471 & 35.0206 & 25.6925 & 20.0000 \\
\hline Crank-Nicolson Scheme & $t_{11}$ & 100.0000 & 72.1100 & 49.3985 & 34.1466 & 25.3213 & 20.0000 \\
\hline
\end{tabular}

The above table clearly shows how close the numerical solutions with both the Schmidt scheme and the CrankNicolson scheme are to the Analytical solution. Shear observation of the above table shows which numerical scheme is closer to the analytical scheme. But the best guide is the result from the absolute relative errors of the two numerical schemes, which is shown in table 7.

Table 7. Absolute Relative Error for the Final Solution of the Heat Equation.

\begin{tabular}{|l|l|l|l|l|l|l|l|}
\hline & & $\boldsymbol{x}_{\mathbf{1}}=\mathbf{0 . 0 0}$ & $\boldsymbol{x}_{\mathbf{2}}=\mathbf{0 . 2 0}$ & $\boldsymbol{x}_{\mathbf{3}}=\mathbf{0 . 4 0}$ & $\boldsymbol{x}_{\mathbf{4}}=\mathbf{0 . 6 0}$ & $\boldsymbol{x}_{\mathbf{5}}=\mathbf{0 . 8 0}$ & $\boldsymbol{x}_{\mathbf{6}}=\mathbf{1 . 0 0}$ \\
\hline Schmidt Scheme & $t_{11}$ & 0.0000 & 0.0090 & 0.0183 & 0.0150 & 0.0307 & 0.1714 \\
\hline Crank-Nicolson Scheme & $t_{11}$ & 0.0000 & 0.0045 & 0.0068 & 0.0103 & 0.0447 & 0.1714 \\
\hline
\end{tabular}

Both schemes' absolute relative error at the boundary points, that is, $x=0$ and $x=1$ are the same. In the range $0.2 \leq x \leq$ 0.6 , the Crank-Nicolson scheme is the best representation of the Analytical solution. However for $x=0.8$, the Schmidt scheme seems to perform better than the Crank-Nicolson scheme.

However on a scale of 100 , the Crank-Nicolson scheme will occupy 75 while the Schmidt scheme will occupy 25. This alone cannot however inform the choice of one over the other because if one considers a particular point alone on the rod, the Schmidt may be better than the Crank-Nicolson scheme.

\section{CONCLUSIONS}

- At all discrete points of the rod $0 \leq x \leq 1$ at the given time frame $0 \leq t \leq 0.1$, the analytical solution fails to conform to the initial conditions of the problem. This is so because the temperature of the rod at the insulated end at time $t=0$ is supposed to be $20^{\circ} \mathrm{C}$ whiles the remaining points were supposed to be very close to the initial temperature with the exception of the end immersed in boiling water.

- Ironically, the temperature at point $x=0.80$ was somehow closer to the room temperature than at the point $x=1.00$. This is surprising since heat transferred from the immersed end of the rod actually reduces as it gets to the insulated end. The analytical method however suggests that within the range $0.80 \leq x \leq 1.00$ temperature was rising instead of falling. There was however exception for the time frame $0.07 \leq t \leq 0.08$ at points $0.80 \leq x \leq 1.00$ on the rod where the behaviour conducting property of the rod was obeyed.

- The Schmidt scheme obeys and conforms perfectly with the initial conditions of the rod, maintaining the initial temperature of $20^{\circ} \mathrm{C}$ at all points of the rod with the exception of the immersed end which assumes the temperature of the boiling water. This presents the Schmidt scheme as a huge improvement of the analytical method.
- The Crank-Nicolson scheme complies well with the initial conditions except at the boundary when it is supposed to be $T(1,0)=20$ but the numerical solution actually gives $0^{\circ} \mathrm{C}$

- Per the Analytical method, the rod's conductivity drops in the range $0.00 \leq x \leq 0.40$ for the time $0.00 \leq t \leq 0.04$ before it rises again. For the Crank-Nicolson scheme, the anomaly in heat conduction is seen at $x=0.08$ in the time interval $0.01 \leq t \leq 0.03$ where as the Schmidt scheme had no anomaly.

- With $r$ specially chosen such that the stability of both the Schmidt and Crank-Nicolson schemes is not compromised, the claim of superiority of one scheme over the other is far fetched. Infact superiority can only be claimed with respect to a particular discrete point in question.

- The Schmidt scheme has shown to be more reliable than the Crank-Nicolson scheme which is strangely seen as an improvement of the Schmidt scheme.

\section{REFERENCES}

[1] Glenn Ledder. 2005. Differential Equations: A Modeling Approach. McGraw-Hill.

[2] John H. Matthews and Kurtis D. Fink 2004. Numerical Methods Using MATLAB. Pearson Education, Inc.

[3] Paul Dawkins. 2007. Lecture Notes on Differential Equations. http://tutorial.math.lamar.edu.

[4] David Richards and Adam Abrahamsen. 2002. The One Dimensional Heat Equation.

[5] Alfio Quarteroni, Riccardo Sacco and Fausto Saleri. 2000. Numerical Mathematics. Springer-Verlag New York, Inc.

[6] Golub, Gene H., and James M. Ortega. 1992. Scientific Computing and Differential Equation: An Introduction to Numerical Methods. San Diego: Academic Press. 